\title{
De-labelling severe asthma diagnosis: the challenge of DIPNECH
}
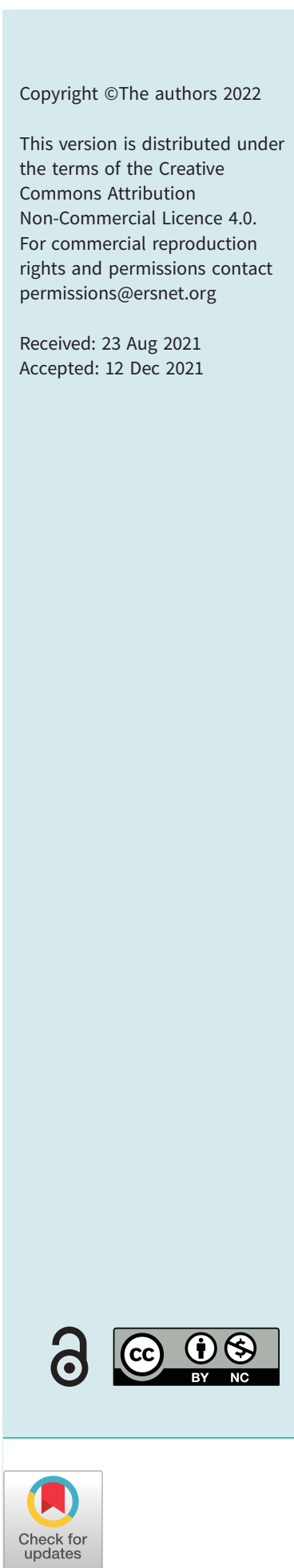

To the Editor:

Diffuse idiopathic pulmonary neuroendocrine cell hyperplasia (DIPNECH) is a rare pulmonary disease characterised by neuroendocrine cell hyperplasia within the bronchial epithelium [1]. The clinical presentation is characterised by nonspecific respiratory symptoms such as chronic cough, dyspnoea and bronchospasm. Given the rarity of the disease and the low specificity of symptoms, the diagnosis of DIPNECH is challenging and the time between symptom onset and diagnosis is long [1]. DIPNECH comprises a generalised proliferation of scattered neuroendocrine cells, small nodules (neuroendocrine bodies) or a linear proliferation of pulmonary neuroendocrine cells. It has been suggested that DIPNECH may mimic [2] or precede [3] asthma. The role of products of neuroendocrine cells such as substance P, which contribute to eosinophil migration, has been suggested to explain asthma symptoms in DIPNECH [3]. However, the characteristics of patients with DIPNECH who have symptoms suggestive of asthma have never been described. The aim of our study was to determine whether patients diagnosed with DIPNECH and initially referred for severe asthma management had specific characteristics.

A retrospective study was conducted in the respiratory medicine departments of four hospitals in France. We reviewed all medical records of patients who were diagnosed with DIPNECH between January 2015 and June 2019. The inclusion criteria were respiratory symptoms and a histological pattern of DIPNECH on surgical lung biopsy. We present here the characteristics of the patients who were referred for management of severe asthma. The study was approved by the institutional review board of the French Society of Respiratory Diseases (Société de Pneumologie de Langue Française: CEPRO 2019-031).

Among 21 patients included in the whole cohort, 20 (95.2\%) were female, with a mean \pm sD age of $62.4 \pm 9.1$ years. All patients in the whole DIPNECH cohort had cough and multiple nodules. Regarding treatment, 15 (71.4\%) were given inhaled steroids.

$10(47.6 \%)$ out of 21 patients were initially referred for severe asthma management. Among those patients, only one patient was male. All the patients had chronic cough and the median (interquartile range (IQR)) duration of cough was 17 (10-30) years. The median (IQR) eosinophil count was 0.18 (0.09$0.33) \times 10^{9}$ cells $\cdot \mathrm{L}^{-1}$ (table 1 ). Four out of 10 patients had an eosinophil count $<0.15 \times 10^{9}$ cells $\cdot \mathrm{L}^{-1}$. The median (IQR) concentration of total IgE was $15(30-98) \mathrm{kIU} \cdot \mathrm{L}^{-1}$. The median (IQR) forced expiratory volume in $1 \mathrm{~s}\left(\mathrm{FEV}_{1}\right)$ was $61 \%$ predicted (35-79\% pred). Regarding radiological features, computed tomography (CT) scans showed multiple nodules in all patients and air trapping in six (54.5\%) patients (figure 1).

The characteristics of our patients were similar to those reported in the literature in terms of gender (female predominance), age and respiratory symptoms [4-6]. In our study, the proportion of patients with DIPNECH who had a prior diagnosis of asthma was $47.6 \%$. This is slightly higher than the previously described prevalence which ranges from $26 \%$ to $40 \%$ [5, 6]. The fact that our centres specialise in severe asthma may explain the higher prevalence. Diagnosing asthma in patients with DIPNECH is very challenging. In fact, respiratory symptoms of DIPNECH are not specific and can mimic asthma. In our study, no specific symptoms were observed in patients with DIPNECH who were referred for severe asthma management compared to patients referred for another reason. Airflow limitation seemed to be a bit more severe, but the difference was not statistically significant. Interestingly, none of the patients with DIPNECH and initially labelled as severe asthma had a history of atopy. Bronchodilator response was noted in only three patients. However, this criterion is not specific to asthma and a recent study in three

Shareable abstract (@ERSpublications)

DIPNECH is a differential diagnosis of severe asthma with no specific biomarkers. Chronic cough and multiple nodules on $\mathrm{CT}$ should prompt clinicians to consider this diagnosis. Differentiating DIPNECH from severe asthma remains crucial. https://bit.ly/3mmFbQn

Cite this article as: Hurabielle C, Taillé C, Prévot G, et al. De-labelling severe asthma diagnosis: the challenge of DIPNECH. ERJ Open Res 2022; 8: 00485-2021 [DOI: 10.1183/23120541.00485-2021]. 
TABLE 1 Characteristics of patients with diffuse idiopathic pulmonary neuroendocrine cell hyperplasia referred for asthma management

\begin{tabular}{|c|c|c|c|c|c|c|c|c|c|c|}
\hline & \multicolumn{10}{|c|}{ Patients } \\
\hline & 1 & 2 & 3 & 4 & 5 & 6 & 7 & 8 & 9 & 10 \\
\hline Age (years) & 68 & 77 & 76 & 56 & 50 & 71 & 65 & 66 & 55 & 62 \\
\hline Gender & $\mathrm{F}$ & $\mathrm{F}$ & $\mathrm{F}$ & $\mathrm{F}$ & $\mathrm{F}$ & $\mathrm{F}$ & $\mathrm{F}$ & M & $\mathrm{F}$ & $\mathrm{F}$ \\
\hline BMI $\left(\mathrm{kg} \cdot \mathrm{m}^{-2}\right)$ & 29.4 & 23.1 & 22.8 & 32.3 & 24.8 & 26.2 & 27.6 & 25.0 & 25.9 & 22.7 \\
\hline History of smoking & No & No & No & Yes & No & No & No & No & No & Yes \\
\hline Dry cough & Yes & Yes & Yes & Yes & Yes & Yes & Yes & Yes & Yes & Yes \\
\hline Cough duration (years) & $>30$ & 10 & 30 & 10 & 25 & 10 & $>30$ & 12 & 14 & 20 \\
\hline$\geqslant 2$ exacerbations within the past 12 months & Yes & No & Yes & No & No & No & Yes & No & No & No \\
\hline Rhinitis & Yes & No & Yes & No & No & No & Yes & No & No & No \\
\hline Dose inhaled steroid ( $\mu \mathrm{g} \cdot \mathrm{day}^{-1}$ of fluticasone equivalent dose) & 2000 & 400 & 800 & 750 & 1000 & 800 & 2000 & 1000 & 800 & 1000 \\
\hline Oral steroids & No & No & No & No & Yes & No & No & No & No & No \\
\hline $\mathrm{FEV}_{1} / \mathrm{FVC}(\%)$ & 68 & 64 & 41 & 79 & 33 & 58 & 57 & 91 & 63 & 73 \\
\hline BHR & Yes & Yes & No & No & No & No & No & No & Yes & No \\
\hline $\mathrm{FEV}_{1}(\mathrm{~L})$ & 1.69 & 1.84 & 0.80 & 0.80 & 0.70 & & 0.88 & 1.20 & 1.83 & 1.73 \\
\hline $\mathrm{FEV}_{1}(\%)$ & 71 & 92 & 36 & 33 & 26 & 70 & 52 & 40 & 74 & 103 \\
\hline TLC (\%) & NA & 108 & 119 & 64 & 121 & 111 & 96 & 64 & 107 & 102 \\
\hline RV (\%) & NA & 132 & 198 & 115 & 229 & 152 & 129 & 106 & 123 & 98 \\
\hline$F_{\mathrm{ENO}}(\mathrm{ppb})$ & 6 & NA & NA & NA & NA & NA & NA & NA & 6 & NA \\
\hline Air trapping & Yes & No & Yes & No & Yes & No & Yes & Yes & Yes & No \\
\hline Number of pulmonary nodules & $>10$ & $2-5$ & $>10$ & $2-5$ & $5-10$ & $2-5$ & $2-5$ & $2-5$ & $2-5$ & $>10$ \\
\hline Blood eosinophils $\left(10^{9}\right.$ cells $\left.\cdot \mathrm{L}^{-1}\right)$ & 0.21 & 0.23 & 0.84 & 0.15 & 0.24 & 0.14 & 0.06 & 0.07 & 0.09 & 0.59 \\
\hline Total IgE $\left(\mathrm{kUI} \cdot \mathrm{L}^{-1}\right)$ & 15 & NA & 22 & 37 & 50 & NA & 15 & NA & 241 & NA \\
\hline Somatostatin analogue & Yes & No & No & No & Yes & No & No & No & No & No \\
\hline Effect on cough & Yes & & & & No & & & & & \\
\hline mTor inhibitor & No & No & Yes & No & Yes & No & No & No & No & No \\
\hline Effect on cough & & & Yes & & No & & & & & \\
\hline
\end{tabular}

Cough was assessed at 6 months after therapy initiation. Cough improvement or no cough change was subjectively collected from patients' testimonies. BMI: body mass index; $\mathrm{FEV}_{1}$ : forced expiratory volume in $1 \mathrm{~s}$; FVC: forced vital capacity; BHR: bronchial hyperresponsiveness; TLC: total lung capacity; RV: residual volume; $F_{\mathrm{ENO}}$ : fractional exhaled nitric oxide; F: female; M: male; NA: not assessed.

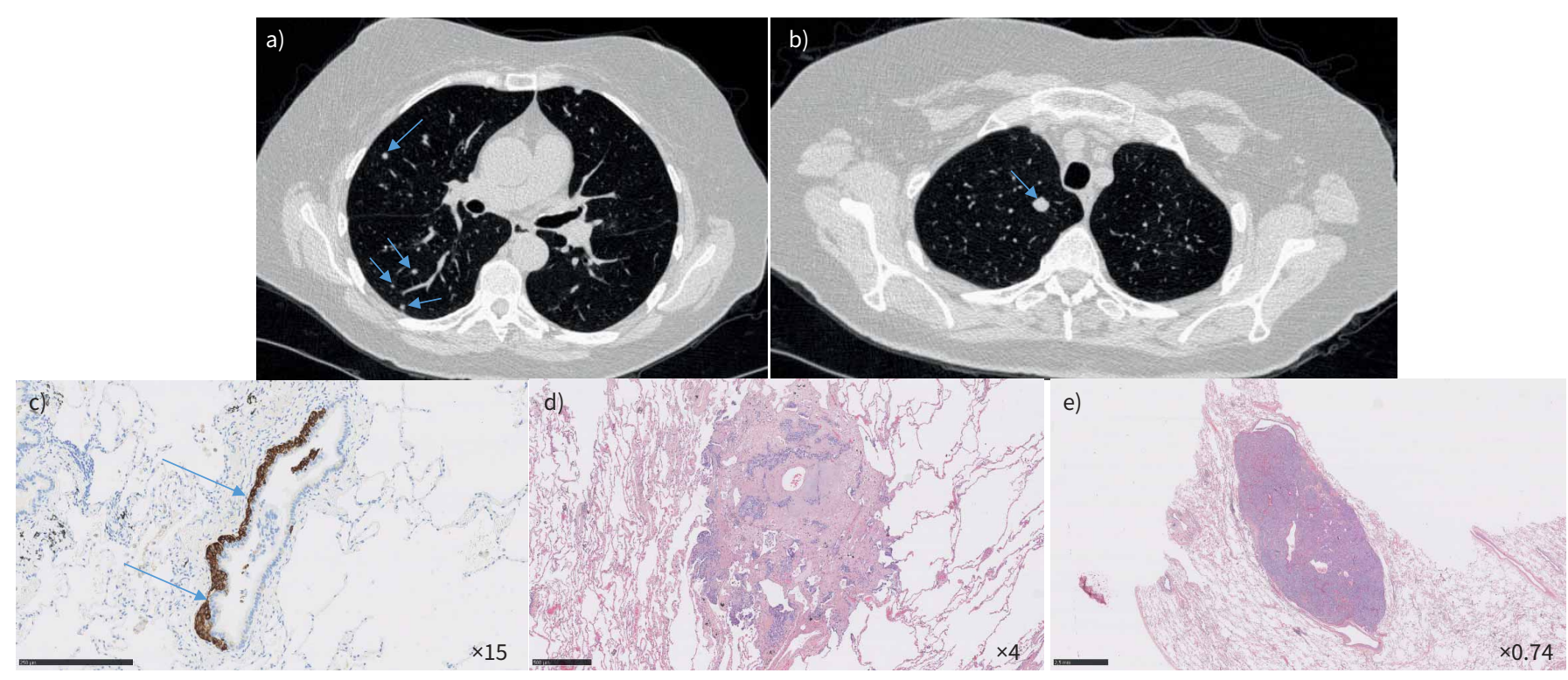

FIGURE 1 Diffuse idiopathic pulmonary neuroendocrine cell hyperplasia in a 68-year-old woman. Chest computed tomography scan showed a) multiple nodules (arrows) and b) an 11-mm nodule in the right upper lobe (arrow). Wedge resection of the right upper lobe showed c) neuroendocrine cell hyperplasia beneath the bronchiolar epithelium (arrows) highlighted by chromogranin-A staining (scale bar=250 $\mu \mathrm{m}$ ); d) a tumourlet (scale bar=500 $\mu \mathrm{m}$ ); and e) a carcinoid tumour (haematoxylin and eosin staining) (scale bar=2.5 $\mathrm{mm}$ ). 
large cohorts concluded that bronchodilator reversibility was at least as common in participants with COPD as those with asthma [7]. Moreover, in a recent study, it has also been shown that bronchial hyperresponsiveness (BHR) is observed in one-third of patients with DIPNECH [8]. In two other studies, $33 \%$ and $10 \%$ of patients with DIPNECH manifested a positive bronchodilator response $[4,6]$. This means that BHR is not restricted to patients with asthma and is common in DIPNECH. Consequently, the presence of BHR in DIPNECH patients does not mean that those patients also have asthma. In our study, no histological pattern of asthma was described on surgical lung biopsies.

Regarding biological markers, there are no specific features. The median blood eosinophil count seems to be low $\left(0.18 \times 10^{9}\right.$ cells $\left.\cdot \mathrm{L}^{-1}\right)$. However, only four out of 10 patients had an eosinophil count $<0.15 \times 10^{9}$ cells $\cdot \mathrm{L}^{-1}$, indicative of non-type- 2 inflammation. In addition, the median concentration of total IgE seems to be low $\left(15 \mathrm{kIU} \cdot \mathrm{L}^{-1}\right)$. In a recent study conducted in a global real-life severe asthma cohort, $1.6 \%$ of patients showed a non-eosinophilic phenotype [9]. This study emphasises that a non-eosinophilic phenotype is likely to be uncommon in severe asthma patients. According to our study and the low eosinophil count observed in our patients with DIPNECH, clinicians should be aware of considering differential diagnosis including DIPNECH in patients labelled as non-eosinophilic severe asthma.

Interestingly, some characteristics are indicative of DIPNECH. In our study, $100 \%$ of the patients with DIPNECH had a chronic nonproductive cough. In other case series, the proportion of patients with cough ranges from $21 \%$ to $71 \%$ [4-6, 10]. In DIPNECH, cough is commonly refractory to standard treatments. In European Respiratory Society guidelines published in 2020, it is not recommended to routinely perform chest CT scan in chronic cough [11]. In fact, chest CT scan has a low impact on chronic cough management [12]. However, in patients with refractory chronic cough and airflow limitation experiencing long duration of cough (>10 years), chest CT scan seems to be an interesting tool for DIPNECH screening. Further studies are needed to better identify those patients.

In severe asthma, although high-resolution CT (HRCT) abnormalities are common [13], HRCT scan does not seem appropriate to differentiate subphenotypes of asthma [14]. However, according to Global Intiative for Asthma guidelines on difficult-to-treat and severe asthma, HRCT should be considered as a tool for screening of comorbidities and differential diagnoses. To the best of our knowledge, no biological or clinical characteristics have been available up to now for the decision to perform HRCT in severe asthma. According to our study, persistent dry cough in patients labelled with severe asthma should prompt clinicians to perform HRCT for the investigation of alternative diagnoses such as DIPNECH. A CT scan is an essential component of the investigation of DIPNECH. In fact, in our study, the presence of multiple pulmonary nodules on CT scan was a criterion and air trapping was observed in half of the patients. In the literature, multiple nodules are very common in patients with DIPNECH and this feature is described in as many as $100 \%$ of the patients in certain case series [10]. In addition, mosaic perfusion has been reported as the predominant finding in several studies. In the context of severe asthma, the combination of multiple nodules and mosaic perfusion on CT scan is highly evocative of DIPNECH.

Histopathological confirmation is required. In clinical practice, distinction of the two diseases is crucial, considering that the treatment for severe asthma and that for DIPNECH are different, in order to avoid inadequate use of biological therapies or continuous oral steroid therapy, for example. In DIPNECH, it has been shown that somatostatin analogues improve respiratory symptoms. In a recent study conducted in 42 patients with DIPNECH treated with somatostain analogues, 15 (36\%) reported mild improvement of symptoms, six (14\%) reported moderate improvement and 11 (26\%) reported significant improvement [15]. Furthermore, somatostatin analogues seem to have a beneficial effect on cough [16]. Cough and dyspnoea improvement have been described in three patients using an mTor inhibitor [17]. In our study, a small number of patients received a somatostatin agonist or an mTor inhibitor and the response was equivocal.

Given the histopathological features and the patients' characteristics (low type 2 inflammation), a coexistence of two rare diseases (severe asthma and DIPNECH) seems to be unlikely. Very low FEV 1 values feature in approximately half of our patients, but, like low type 2 inflammation, this feature is uncommon in severe asthma. In patients with DIPNECH, low $\mathrm{FEV}_{1}$ values could be the expression of constrictive bronchiolitis. In a recent study, 52\% of patients with DIPNECH had airflow limitation [8]. However, only 53\% of those patients demonstrated constrictive bronchiolitis on histological examination. The proportion of patients with constrictive bronchiolitis was similar between patients with and without airflow limitation (53\% versus 44\%, respectively). The authors suggest that pathophysiological mechanisms other than constrictive bronchiolitis contribute to airflow obstruction in DIPNECH. FEV 1 variability, included in asthma definitions, was not observed in our cohort. Our series does not support the 
hypothesis of DIPNECH-induced asthma, but rather highlights the fact that DIPNECH should be considered more as a differential diagnosis of severe asthma than a comorbid condition of severe asthma.

Our study has limitations. The number of patients is low, but DIPNECH is a very rare disease and our cohort is among the largest in the literature. We show that patients with DIPNECH who were referred for uncontrolled asthma management have no specific usual biomarkers. However, we cannot exclude a recruitment bias related to the investigators' specific involvement in severe asthma care.

To conclude, DIPNECH clearly appears to be a differential diagnosis of severe asthma, particularly in women. The presence of chronic cough with a long duration and multiple nodules on the CT scan should prompt clinicians to consider this differential diagnosis. Given the difference in prognosis and treatment, differentiating DIPNECH and severe asthma remains crucial to improve patient outcomes.

\section{Caroline Hurabielle ${ }^{1}$, Camille Taillé ${ }^{2}$, Grégoire Prévot ${ }^{1}$, Maud Russier ${ }^{3}$, Alain Didier ${ }^{1,4}$, Pierre-Olivier Girodet ${ }^{5}$, Magali Colombat ${ }^{6}$, Julien Mazières ${ }^{1}$ and Laurent Guilleminault ${ }^{1,4}$}

${ }^{1}$ Dept of Respiratory Medicine, Toulouse University Hospital Centre, Toulouse, France. ${ }^{2}$ Groupe Hospitalier Universitaire AP-HP Nord-Université de Paris, Hôpital Bichat, Service de Pneumologie et Centre de Référence Constitutif des Maladies Pulmonaires Rares; Inserm UMR 1152; CRISALIS F-CRIN, Paris, France. ${ }^{3}$ Centre Hospitalier Régional d'Orléans, Orléans, France. ${ }^{4}$ Center for Pathophysiology of Toulouse Purpan, INSERM U1043, CNRS UMR 5282, Université Toulouse III; CRISALIS F-CRIN, Toulouse, France. ${ }^{5}$ Université de Bordeaux, Pharmacologie, CIC1401, INSERM U1045, F-33000; CRISALIS F-CRIN, Bordeaux, France. ${ }^{6}$ Service d'anatomopathologie, $\mathrm{CHU}$ de Toulouse, Toulouse France.

Corresponding author: Laurent Guilleminault (guilleminault.l@chu-toulouse.fr)

Provenance: Submitted article, peer reviewed.

Conflict of interest: C. Hurabielle has nothing to disclose. C. Taillé reports grants, personal fees and nonfinancial support from GSK, Sanofi and AstraZeneca, personal fees from Novartis and Stallergenes, and participation on a data safety monitoring or advisory board for AstraZeneca, GSK, Sanofi and Novartis. G. Prévot has nothing to disclose. M. Russier has nothing to disclose. A. Didier has nothing to disclose. P-O. Girodet has nothing to disclose. M. Colombat has nothing to disclose. J. Mazières has nothing to disclose. L. Guilleminault has nothing to disclose.

\section{References}

1 Rossi G, Cavazza A, Spagnolo P, et al. Diffuse idiopathic pulmonary neuroendocrine cell hyperplasia syndrome. Eur Respir J 2016; 47: 1829-1841.

2 Patel JD, Kerby G. Diffuse idiopathic pulmonary neuroendocrine hyperplasia mimicking uncontrolled asthma. Am J Respir Crit Care Med 2010; 181: A5873.

3 Sanaee MS, O'Byrne PM, Nair P. Diffuse idiopathic pulmonary neuroendocrine hyperplasia, chronic eosinophilic pneumonia, and asthma. Eur Respir J 2009; 34: 1489-1492.

4 Nassar AA, Jaroszewski DE, Helmers RA, et al. Diffuse idiopathic pulmonary neuroendocrine cell hyperplasia: a systematic overview. Am J Respir Crit Care Med 2011; 184: 8-16.

5 Davies SJ, Gosney JR, Hansell DM, et al. Diffuse idiopathic pulmonary neuroendocrine cell hyperplasia: an under-recognised spectrum of disease. Thorax 2007; 62: 248-252.

6 Carr LL, Chung JH, Duarte Achcar R, et al. The clinical course of diffuse idiopathic pulmonary neuroendocrine cell hyperplasia. Chest 2015; 147: 415-422.

7 Janson C, Malinovschi A, Amaral AF, et al. Bronchodilator reversibility in asthma and COPD: findings from three large population studies. Eur Respir J 2019; 54: 1900561.

8 Samhouri BF, Azadeh N, Halfdanarson TR, et al. Constrictive bronchiolitis in diffuse idiopathic pulmonary neuroendocrine cell hyperplasia. ERJ Open Res 2020; 6: 00527-2020.

9 Heaney LG, de Llano L P, Al-Ahmad M, et al. Eosinophilic and noneosinophilic asthma: an expert consensus framework to characterize phenotypes in a global real-life severe asthma cohort. Chest 2021; 160: 814-830.

10 Trisolini R, Valentini I, Tinelli C, et al. DIPNECH: association between histopathology and clinical presentation. Lung 2016; 194: 243-247.

11 Morice AH, Millqvist E, Bieksiene K, et al. ERS guidelines on the diagnosis and treatment of chronic cough in adults and children. Eur Respir J 2020; 55: 1901136.

12 Descazeaux M, Brouquières D, Didier A, et al. Impact of chest computed tomography scan on the management of patients with chronic cough. ERJ Open Res 2021; 7: 00222-2021. 
13 Gupta S, Siddiqui S, Haldar P, et al. Qualitative analysis of high-resolution CT scans in severe asthma. Chest 2009; 136: 1521-1528.

14 Gupta S, Siddiqui S, Haldar P, et al. Quantitative analysis of high-resolution computed tomography scans in severe asthma subphenotypes. Thorax 2010; 65: 775-781.

15 Al-Toubah T, Strosberg J, Halfdanarson TR, et al. Somatostatin analogs improve respiratory symptoms in patients with diffuse idiopathic neuroendocrine cell hyperplasia. Chest 2020; 158: 401-405.

16 Chauhan A, Ramirez RA. Diffuse idiopathic pulmonary neuroendocrine cell hyperplasia (DIPNECH) and the role of somatostatin analogs: a case series. Lung 2015; 193: 653-657.

17 Russier M, Plantier L, Derot G, et al. Diffuse idiopathic pulmonary neuroendocrine cell hyperplasia syndrome treated with sirolimus. Ann Intern Med 2018; 169: 197-198. 